\title{
Interferência da dupla tarefa no desempenho da marcha em indivíduos com doença de Parkinson
}

\author{
Dual task interference on gait performance in \\ individuals with Parkinson Disease
}

\author{
Felipe Roberto de Araújo' ${ }^{1}$ (1) \\ Denise S. de Araújo 2 (1) \\ Camila Lobo de Aguiar Gomes 3 (1) \\ Ana Loyse de Souza Medeiros 4 (1) \\ Afonson Luiz Medeiros Gondim 5 (1) \\ Roberta Oliveira Cacho ${ }^{6}$ (i) \\ Enio Walker Azevedo Cacho7 ${ }^{7}$
}

\begin{abstract}
${ }^{1}$ Autor para correspondência. Universidade Federal do Rio Grande do Norte (Santa Cruz). Rio Grande do Norte, Brasil. felipe.ojuara@hotmail.com ${ }^{2-7}$ Universidade Federal do Rio Grande do Norte (Santa Cruz). Rio Grande do Norte, Brasil. denisearaujo.s@hotmail.com, camilalobofisio@gmail.com, loysemedeiros26@gmail.com, afonsongondim2014.1@hotmail.com, ro_fisio1@hotmail.com, eniowalker@gmail.com
\end{abstract}

RESUMO | INTRODUÇÃO: A realização de duas tarefas de forma simultânea exige a capacidade de concentrar atenção e executar duas tarefas ao mesmo tempo. Em indivíduos com Doença de Parkinson as interferências podem ser ainda maiores, visto que esses indivíduos necessitam de um grau maior de atenção apenas para executar uma tarefa única, como no caso, a marcha. OBJETIVO: Analisar a interferência da dupla tarefa na marcha de pacientes com DP. MATERIAIS E MÉTODOS: Estudo observacional de caráter transversal, de natureza quantitativa. Cinco indivíduos com Doença de Parkinson, de ambos os sexos, participaram do grupo experimental; e cinco indivíduos saudáveis fizeram parte do grupo controle. Os participantes tiveram a marcha avaliada com e sem interferência cognitiva, utilizando: Escala de Equilíbrio de Berg; Índice Dinâmico da Marcha; teste de mobilidade funcional (Time Up and Go Test), Esteira Ergométrica e o Stroop Test adaptado para analfabetos. RESULTADOS: Quando comparados Grupo Experimental e Grupo Controle foram encontradas diferenças estatisticamente significantes nas condições de marcha com interferência de dupla tarefa (Dupla Tarefa Número e Dupla Tarefa Cor) nas variáveis de quantidade de acertos e amplitude de movimento. CONCLUSÃO: A dupla tarefa em pacientes com DP, interfere principalmente na função cognitiva, enquanto a função motora da marcha permanece parcialmente preservada.
ABSTRACT | BACKGROUND: Performing dual tasks simultaneously requires the ability to focus attention and perform two activities at the same time. In individuals with Parkinson's disease, the interferences may be greater, as these individuals require a major degree of attention just to perform a single task, as in this case, walking. OBJECTIVE: To analyze the interference of dual task in the gait of PD individuals. MATERIAL AND METHODS: Observational cross-sectional study with a prospective structure of a quantitative nature. Five individuals with Parkinson Disease, of both gender, participated in the experimental group; five healthy individuals were part of the control group. The participants had their gait assessed with and without cognitive interference, using: Berg's Balance Scale; Dynamic Gait Index; functional mobility test (Time Up and Go Test), Treadmill gait and Stroop Test adapted for illiterates. RESULTS: When comparing the Experimental Group and the Control Group, statistically significant differences were found in the gait conditions with dual task interference (Dual Task Number and Dual Task Color) in the variables of number of correct answers and range of motion. CONCLUSION: The dual task in PD patients mainly interferes with cognitive function, while the motor function of gait remains partially preserved.

KEYWORDS: Parkinson disease. Gait. Dual task.

PALAVRAS-CHAVE: Doença de Parkinson. Marcha. Dupla tarefa. 


\section{Introdução}

A doença de Parkinson (DP) afeta entre 1 a $2 \%$ das pessoas acima de 65 anos no mundo, e com prevalência de $3 \%$ no Brasil'. Os distúrbios da marcha na DP estão entre os principais sintomas motores e um dos mais incapacitantes, causadas pela bradicinesia, rigidez, tremor e reflexos posturais prejudicados, e que geralmente é como marcha festinada².

A marcha é um processo dinâmico, controlado pelo córtex, tronco cerebral e medula espinhal, onde qualquer deficiência em um destes sistemas pode afetar o seu controle e regulação ${ }^{3}$. Em especial, nas adaptações que estes sistemas têm que produzir durante as restrições ambientais presente durante uma caminhada, o que pode torná-la insegura e incapacitante para indivíduos com DP4.

Muitas são as propostas terapêuticas utilizadas na reabilitação da marcha em indivíduos com DP, sendo uma delas a utilização de pistas externas (visuais) ${ }^{5}$. As abordagens terapêuticas tem o intuito de melhorar os parâmetros da marcha, incluindo velocidade, cadência e comprimento da passada, entre outros.

Apesar da marcha ser uma atividade predominantemente automática, nos pacientes com DP, ela requer um grau maior de atenção ${ }^{6}$. Assim, a realização de duas tarefas simultaneamente é influenciado pela capacidade de efetivamente concentrar a atenção em estímulos simultâneos ${ }^{7}$. De acordo com estudos prévios, isto pode ser explicado, pelo fato dos indivíduos com Doença de Parkinson apresentarem um comprometimento sensóriomotor que dificulta o controle automático, fazendo com que necessitem de um maior controle atencional durante a realização de suas atividades motoras diárias. Frequentemente, o efeito da dupla tarefa é mesurado como um indicador de automação do controle motor ${ }^{8,9}$.

A dupla tarefa pode causar prejuízos ao desempenho da marcha, tendo em vista que em estudos anteriores observou-se que em condições de tarefas simultâneas, a demanda atencional é divida e os recursos necessários para compensar a automação da marcha não são totalmente eficazes, deixando prejudicada a locomoção efetiva ${ }^{10}$.

Desta forma, nota-se a importância de se trabalhar atividades terapêuticas que foquem a prática da dupla tarefa, isto é, na utilização de práticas que produzam a interferência cognitiva e/ou motora de uma atividade, sobre outra. Prática esta, realizada em ambiente controlado, a fim de evitar quedas e lesões, mas que produzam uma disputa pela demanda atencional e que seja possível transferir as habilidades aprendidas para as condições ambientais do paciente. No entanto, algumas perguntas ainda precisam ser feitas a respeito do tipo de interferência que deve ser ofertada durante a prática da dupla tarefa e quais seus efeitos.

Alguns estudos vêm produzindo resultados que tentam esclarecer as dúvidas relacionadas ao tema utilizando-se de testes pré-estruturados, como o Stroop Test (ST) e o Simbol Test, e demonstram um aumento na variabilidade da marcha, quando uma tarefa cognitiva é proposta simultaneamente ${ }^{11}$. Diante disso, o objetivo do presente estudo foi analisar os efeitos da dupla tarefa na marcha em esteira de pacientes com DP.

\section{Materiais e métodos}

Trata-se de um estudo observacional, de caráter transversal de natureza predominantemente quantitativa, com amostra por conveniência, realizado de janeiro de 2018 a outubro de 2019, no Laboratório de motricidade e fisiologia humana e no Ambulatório de Fisioterapia da Clínica Integrada da Faculdade de Ciências da Saúde do Trairi - Facisa/UFRN e aprovado pelo Comitê de Ética em Pesquisa (CEP) da instituição sob o parecer CAAE 2.715.132 (CAAE 89527318.3.0000.5568).

\section{Participantes}

O estudo foi dividido em duas etapas: inicialmente foi realizada a triagem dos pacientes com DP, e em seguida, a seleção e avaliação dos indivíduos (que atendessem aos critérios de elegibilidade). A triagem dos pacientes aconteceu a partir dos dados coletados na lista de pacientes da instituição e por meio do contato telefônico, obtiveram-se informações sobre a idade, diagnóstico clínico e funcional, interesse e disponibilidade para participar do estudo. Em seguida, foram recrutados indivíduos controles da comunidade, de forma, a parear com o grupo de paciente com DP.

Desta forma, o estudo foi constituído por dois grupos: O Grupo Experimental (GE), que foi composto de indivíduos com diagnóstico clínico de DP, todos de acordo com um parecer médico de um neurologista, 
classificados pela escala modificada de estadiamento de Hoenh \& Yahr, e que faziam uso contínuo de medicação para DP; e o Grupo Controle (GC), formado de indivíduos saudáveis sem qualquer outra doença neurológica, recrutados da comunidade.

Para ambos os grupos, os indivíduos deveriam apresentar pontuação maior que 24, para escolarizados e 14, para não escolarizados no Mine Exame do Estado Mental (MEEM), além de idade superior a 40 anos. Ambos os grupos aceitaram a participação na pesquisa e assinaram o termo de consentimento livre e esclarecido - TCLE. Foram excluídos do estudo os indivíduos que apresentaram deformidades articulares, artrite ou dores severas, ou outro distúrbio neurológico concomitante a DP (específico ao GE) e que se ausentaram durante o período da pesquisa.

A amostra do estudo foi composta inicialmente de 11 indivíduos, de ambos os sexos, sendo seis do GE e cinco do GC (Figura 1). Um (01) indivíduo foi incapaz de participar do estudo devido a questões de saúde, restando desta forma cinco indivíduos no GE. Já no GC, os cinco indivíduos inicialmente elegíveis, concordaram em participar de todas as etapas do estudo.

Figura 1. Fluxograma da seleção de amostra

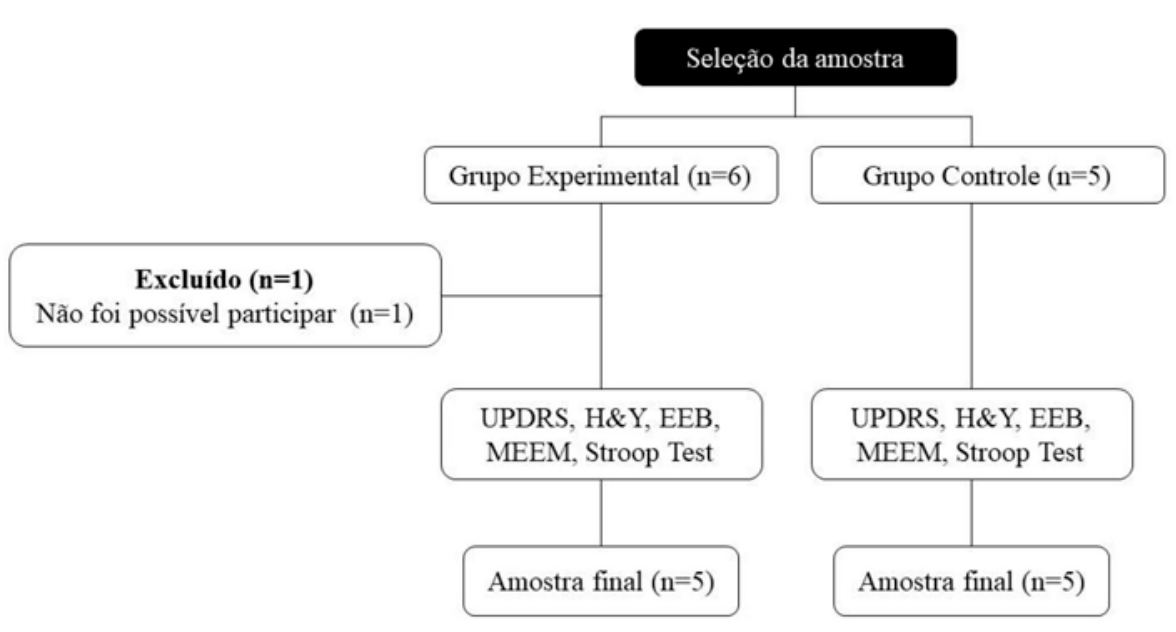

\section{Procedimentos}

Para caracterização da amostra e utilização dos critérios de elegibilidade foram utilizados os seguintes instrumentos clínicos: O Mini Exame de Estado Mental (MEEM), que permite a avaliação da função cognitiva, e possui escore total 30 pontos.

A Escala Unificada de Avaliação para Doença de Parkinson (UPDRS) documenta os efeitos gerais da DP, apresenta-se em quatro domínios que avaliam a progressão da doença de acordo com suas características clínicas e os efeitos das flutuações relacionadas ao uso de fármacos, com uma pontuação máxima de 180 pontos, a pontuação em cada item varia de 0 a 4, sendo que quanto maior a pontuação maior gravidade da doença ${ }^{12}$. Foram utilizados dois domínios da UPDRS, o domínio de atividades de vida diária e do exame motor.

A Escala de Hoehn \& Yahr (H\&Y) modificada que mensura o estado de gravidade da doença em oito etapas onde o estágio zero (0) significa sem sinal da doença e o estágio cinco (5), o paciente encontra-se em cadeira de rodas ou acamado, incapacitado de realizar atividades diárias a menos que seja ajudado ${ }^{13}$.

A Escala de Equilíbrio de Berg (EEB) é um instrumento que mensura o equilíbrio funcional, estando constituída de 14 itens. Para cada item a pontuação varia entre zero (0) e quatro (4) pontos, sendo o escore máximo 56 pontos. 
A pontuação varia de acordo com o nível de dependência para a realização da tarefa. Pontuações entre zero (0) e vinte (20) pontos correspondem a restrição em cadeira de rodas, entre 21 e 40 pontos referem-se à assistência durante a marcha, e 41 a 56 pontos correspondem a independência ${ }^{14}$.

O teste de mobilidade funcional (Time Up and Go Test TUG) é caracterizado por uma tarefa, que se inicia na posição sentada em uma cadeira padrão com braços, o indivíduo mantém os braços apoiados na cadeira, levanta e caminha 3 metros, vira, retorna para a cadeira e senta-se novamente. É registrado o tempo gasto para a realização do teste por completo, sendo considerado até 10 segundos um tempo normal para adultos saudáveis, independentes e sem risco de quedas; valores entre 11-20 segundos são esperados para idosos com deficiência ou frágeis, com independência parcial e baixo risco de quedas; acima de 20 segundos sugere que o idoso apresenta déficit importante da mobilidade física e risco de quedas ${ }^{15}$.

O Índice Dinâmico da Marcha (DGI) tem o objetivo de avaliar e documentar a capacidade do paciente de modificar o padrão de marcha em resposta às mudanças ambientais. O DGI é constituído de oito tarefas que envolvem a marcha em diferentes contextos sensoriais, que incluem superfície plana, mudanças na velocidade da marcha, movimentos horizontais e verticais da cabeça, passar por cima e contornar obstáculos, giro sobre seu próprio eixo corporal, subir e descer escadas. 0 valor máximo corresponde a 24 pontos e valores iguais ou abaixo 19 pontos indica instabilidade na marcha e maior risco de quedas ${ }^{16}$.

\section{Avaliação}

Após o processo de alocação (seleção) e caracterização, foram iniciados os protocolos experimentais para os dois grupos. Foram utilizadas quatro condições experimentais de 20 segundos, cada: Condição 1, marcha em esteira sem dupla tarefa (SDT) (Figura 2A); Condição 2, marcha em esteira com DT Número (cDTN) (o paciente deveria caminhar na esteira enquanto falava uma sequência de 30 itens (números) projetados em tinta preta pelo aparelho de multimídia, em um telão $(1,50$ metros $\times 1,50$ metros) posicionado em frente ao paciente, em uma ordem aleatória que variava do número $1(\mathrm{um})$ ao 11) (Figura 2B); Condição 3, marcha com DT Cor (CDTC) (o paciente deveria caminhar na esteira enquanto falava uma sequência de 30 itens (cores) projetadas pelo aparelho de multimídia em uma ordem aleatória que variava entre 11 cores (vermelho, azul céu, azul anil, marrom, roxo, amarelo, laranja, verde, preto, rosa e cinza)) (Figura 2C); Condição 4, marcha com DT Cor-Número (cDTCN) (o paciente deveria caminhar na esteira enquanto falava uma sequência de 30 itens (números) projetados em tinta colorida no aparelho de multimídia em uma ordem aleatória que variava do número 1 (um) ao 11, apresentados em 11 cores (vermelho, azul céu, azul anil, marrom, roxo, amarelo, laranja, verde, preto, rosa e cinza) (Figura 2D).

Figura 2. Condições de marcha em esteira associada e não associada à tarefa dupla. Figura 2A - marcha sem dupla tarefa (sDT). Figura 2B - marcha com DT número (cDTN). Figura 2C - marcha com DT cor (CDTC). Figura 2D - marcha com DT cor-número (cDTCN)

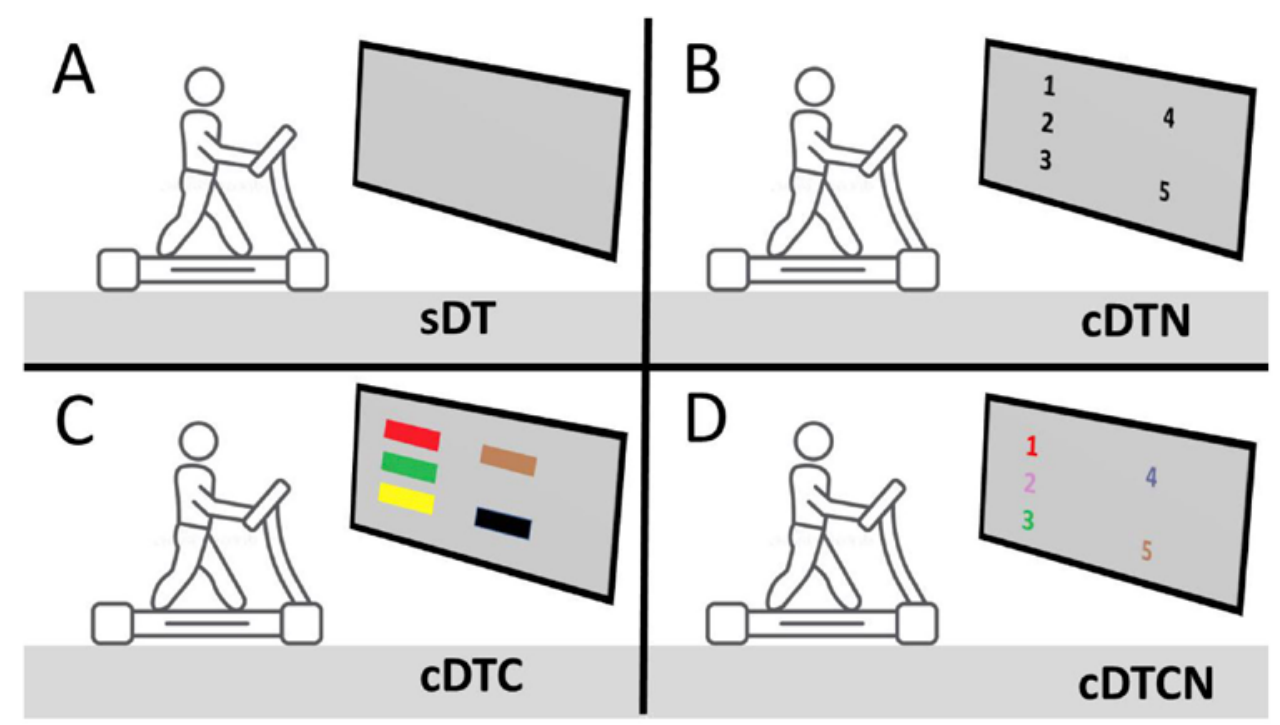


A tarefa cognitiva do protocolo experimental, descrito acima, foi baseado nas atividades cognitivas do Stroop Test (ST) adaptado para analfabeto ${ }^{17}$ que igualmente ao original é apresentado em três partes, sendo que as palavras são substituídas por números.

Para cada parte do teste, foi utilizado um total de 30 itens (números pretos e coloridos e cores), e ao participante foi determinado um tempo de 20 segundos para a execução de cada parte, aconselhando-o a realizar a tarefa o mais rápido possível. Isto é, eles deveriam identificar o número e/ou a cor, e falar corretamente. Desta forma, foi computada variável de número total de acertos (NaST) para cada condição do Stroop Test (cDTN, cDTC e cDTCN).

As variáveis angulares (amplitude total, amplitude máxima e amplitude mínima do joelho direito), foram coletadas para uma melhor compreensão das mudanças na marcha durante as condições experimentais do estudo nos dois grupos. Essas variáveis foram coletadas, por meio de uma câmera Full Hd da marca Canon Vixia R800, posicionada lateralmente a esteira, em uma frequência de amostragem de $30 \mathrm{~Hz}$. Foram utilizados três marcadores com diâmetros de 1,1 cm cada, fixados na perna direita do participante (sobre o trocanter maior do fêmur, o côndilo lateral da tíbia e o maléolo lateral da tíbia).

Após a coleta, os dados foram armazenados em um computador e logo após tratados e processados pelos Softwares CVMob - Versão 4.0 alpha (versão livre). As coordenadas articulares de movimento foram analisadas no plano sagital, considerando 10 ciclos da marcha, o que totalizava 10 passadas. A extensão do joelho foi interpretada neste estudo como um valor próximo a $180^{\circ}$ de amplitude articular e a flexão como um valor menor. A amplitude de movimento do joelho direito foi calculada a partir da diferença entre a amplitude máxima e a mínima. Os valores das variáveis angulares do joelho direito foram calculados a partir da média obtida nas 10 passadas.

Os números e cores foram apresentados por meio de um projetor portátil Dell ${ }^{\circ}$ Resolução nativa: WXGA (1.280 X 800) Cores exibidas em 1,073 bilhão. A Esteira Ergométrica utilizada no protocolo experimental foi da marca EMG System do Brasil Equipamentos Ltda. ®. A velocidade utilizada por cada indivíduo foi ajustada de acordo com o paciente e cada condição foi repetida três vezes para se obter uma média simples.

Os efeitos da dupla tarefa sobre a marcha em esteira nas diversas condições experimentais do estudo foram analisados utilizando-se de medidas relativas (Efeito da Dupla Tarefa - EDT), calculadas a partir de cada variável do estudo (amplitude de movimento, amplitude máxima e mínima de movimento), em uma condição de dupla tarefa e em condição de tarefa única. Um decréscimo sob condições de dupla tarefa (isto é, um custo de dupla tarefa) foi representado por um valor negativo. Uma melhoria em condições de dupla tarefa (isto é, um benefício de dupla tarefa) foi representada por um valor positivo (Eq. 1 1$)^{18}$.

\section{EDT $\{\%\}=$ Variável de tarefa dupla - variável de tarefa única $\times 100$ \\ Variável de tarefa única}

Para variáveis nas quais os valores mais altos indicam desempenho pior (em vez de melhor) (exemplo, amplitude articular do joelho), um sinal negativo foi inserido na fórmula (Eq. 2).

EDT $\{\%\}=-($ Variável de tarefa dupla-Variável de tarefa única) $\times 100$

Variável de tarefa única 


\section{Análise Estatística}

Todos os dados foram armazenados e analisados por meio do programa BioEstat 5.0. Os dados demográficos foram analisados por meio de uma análise descritiva. Para avaliar a normalidade da amostra foi utilizado o teste de Shapiro-Wilk, sendo adotados testes não paramétricos. Para se comparar o número de acertos do teste cognitivo (NaST) durante a marcha em esteira nas três condições (cDTN, cDTC e cDTCN) foi realizado o teste de Friedman para a análise intra grupo, e o teste de Mann-Whitney na análise inter grupo.

E com o intuito de observar o efeito da tarefa cognitiva (EDT) na marcha em esteira nas condições sem (sDT) e com interferência cognitiva (cDTN, CDTC e cDTCN), foram utilizados os mesmos testes, para a avaliação intra grupo (teste de Friedman) e inter grupo (teste de Mann-Whitney). Para todas as análises utilizou-se o nível de significância $p<0.05$.

\section{Resultados}

O estudo contou com uma amostra de 10 indivíduos, sendo cinco do GE e cinco do GC, cada grupo foi composto por três mulheres (60\%) e dois homens (40\%). A table 1 apresenta as demais características demográficas e clínicas dos dois grupos estudados.

Tabela 1. Caracterização dos participantes

\begin{tabular}{ccc}
\hline Variáveis & GRUPO EXPERIMENTAL & GRUPO CONTROLE \\
\cline { 2 - 3 } & Mediana $\left(1^{\circ} \mathrm{Q} / 3^{\circ} \mathrm{Q}\right)$ & Mediana $\left(1^{\circ} \mathrm{Q} / 3^{\circ} \mathrm{Q}\right)$ \\
\hline Idade (anos) & $67.00(53.00 / 67.00)$ & $63.00(53.00 / 67.00)$ \\
Altura $(\mathbf{m})$ & $1.56(1.56 / 1.61)$ & $1.60(1.56 / 1.63)$ \\
Peso $(\mathbf{k g})$ & $66.80(64.00 / 69.00)$ & $71.00(68.00 / 71.50)$ \\
Imc & $26.30(23.30 / 28.90)$ & $1.00(1.00 / 5.00)$ \\
Escolaridade (anos) & $4.00(0.00 / 5.00)$ & - \\
UPDRS & $53.00(26.00 / 66.00)$ & - \\
H\&Y & $1.00(1.00 / 2.50)$ & $56.00(56.00 / 56.00)$ \\
EEB & $52.00(50.00 / 54.00)$ & $7.12(7.03 / 7.32)$ \\
TUG (TEMPO) & $15.43(14.79 / 15.74)$ & $24.00(24.00 / 24.00)$ \\
DGI & $20.00(20.00 / 21.00)$ & \\
\hline
\end{tabular}

UPDRS, Escala Unificada de Avaliação para Doença de Parkinson; H\&Y, Escala de Hoehn \& Yahr modificada; EEB, Escala de Equilíbrio de Berg; TUG, Time Up and Go Teste; DGI, Índice Dinâmico da Marcha. *, diferença significante entre o Grupo Controle e o Grupo Experimental $(p<0.05)$.

De acordo com os dados apresentados na tabela 1, a mediana da Escala H\&Y caracteriza os participantes com DP no estágio inicial da doença e os resultados da EEB indicam que estes indivíduos são independentes. Além disso, os dados referentes ao TUG determinam que estes participantes são idosos frágeis ou dependentes. Por fim, os escores da DGI, mostraram uma ausência de risco de queda nesses idosos com DP. Desta forma, mesmo tratando-se de um grupo de indivíduos no estado inicial da DP, observa-se uma diferença entre os escores quando comparados com os idosos saudáveis do grupo controle, sobretudo no TUG tempo, onde apresentaram maior diferença.

Os dados demográficos não demonstraram diferenças estatísticas significantes entre os dois grupos, assim como o equilíbrio mensurado pela EEB. Diferentemente dos demais dados clínicos, mensurados pela TUG e DGI, onde podemos observar uma diferença estatística significante entre os grupos estudados, com o GC apresentando melhores resultados. 
Em relação aos dados relativos ao NaST durante a marcha em esteira, realizadas nas três condições, podemos observar na tabela 2, que apenas dentro do GC existiram diferenças significantes entre as condições. Foram observadas também, diferenças significativas entre o GC e o GE, nas duas primeiras condições (CDTN e cDTC).

Tabela 2. Dados relativos ao número de acertos (NaST) na tarefa cognitiva nos dois grupos

\begin{tabular}{rcc}
\hline \multirow{2}{*}{ Condições/NaST } & GRUPO EXPERIMENTAL & GRUPO CONTROLE \\
\cline { 2 - 3 } & MEDIANA(1Q/3Q) & MEDIANA(1Q/3Q) \\
\hline CDTN & $17.0(16.0 / 24.0)^{\mathrm{a}}$ & $28.0(28.0 / 29.0)^{\mathrm{b}}$ \\
\hline cDTC & $13.0(12.0 / 15.0)^{\mathrm{c}}$ & $19.0(18.0 / 29.0)$ \\
\hline cDTCN & $14.0(13.0 / 15.0)$ & $19.0(17.0 / 21.0)$
\end{tabular}

GE, Grupo Experimental; GC, Grupo Controle; NaST, número total de acertos; cDTN, com dupla tarefa (stroop test número); cDTC, com dupla tarefa (stroop test cor); CDTCN, com dupla tarefa (stroop cor número). a , diferença estatisticamente significante na CDTN, entre o GE e o GC; ${ }^{b}$, diferença estatisticamente significante entre a CDTN e cDTCN no GC; ${ }^{c}$, diferença estatisticamente significante na cDTC, entre o GE e o GC; $(p<0.05)$.

Na tabela 3, podemos observar a presença de diferenças significativas entre o GC e GE, na amplitude de movimento, nas condições CDTN e cDTC. Não foram encontradas diferenças estatísticas entre as várias condições no GE.

Tabela 3. Variáveis funcionais e articulares da marcha em esteira e o efeito da dupla tarefa

\begin{tabular}{|c|c|c|c|c|c|c|c|}
\hline \multirow{3}{*}{ Variáveis/Condições } & \multicolumn{7}{|c|}{ GRUPO CONTROLE } \\
\hline & sDT & \multicolumn{2}{|l|}{ cDTN } & \multicolumn{2}{|l|}{ cDTC } & \multicolumn{2}{|l|}{ cDTCN } \\
\hline & $\begin{array}{l}\text { Valores da } \\
\text { tarefa única }\end{array}$ & \multicolumn{2}{|c|}{$\begin{array}{c}\text { Valores da tarefa } \\
\text { dupla/Valores da EDT } \\
(\%)\end{array}$} & \multicolumn{2}{|c|}{$\begin{array}{c}\text { Valores da tarefa } \\
\text { dupla/Valores da EDT } \\
(\%)\end{array}$} & \multicolumn{2}{|c|}{$\begin{array}{c}\text { Valores da tarefa } \\
\text { dupla/Valores da EDT } \\
(\%)\end{array}$} \\
\hline Amplitude & $47.22 \pm 17.60$ & $47.62 \pm 16.65$ & $0.84^{\mathrm{a}}$ & $43.87 \pm 11.70$ & $-7.09^{b}$ & $49.54 \pm 3.47$ & 4.91 \\
\hline Amplitude Máxima & $179.35 \pm 0.62$ & $179.30 \pm 1.10$ & -0.02 & $179.48 \pm 0.55$ & 0.07 & $179.68 \pm 0.13$ & 0.18 \\
\hline \multirow[t]{3}{*}{$\begin{array}{l}\text { Amplitude } \\
\text { Mínima }\end{array}$} & $132.13 \pm 16.75$ & $131.67 \pm 15.56$ & -0.34 & $135.60 \pm 11.17$ & 2.62 & $130.13 \pm 3.51$ & -1.51 \\
\hline & \multicolumn{7}{|c|}{ GRUPO EXPERIMENTAL } \\
\hline & sDT & cDTN & & CDTC & & CDTCN & \\
\hline Amplitude & $50.65 \pm 9.60$ & $45.67 \pm 10.69$ & $-9.83^{a}$ & $46.02 \pm 14.43$ & $-9.14^{b}$ & $46.20 \pm 12.32$ & -8.78 \\
\hline Amplitude Máxima & $176.13 \pm 5.63$ & $176.19 \pm 4.65$ & 0.03 & $176.35 \pm 1.98$ & 0.12 & $179.62 \pm 0.51$ & 1.98 \\
\hline $\begin{array}{l}\text { Amplitude } \\
\text { Mínima }\end{array}$ & $125.47 \pm 10.66$ & $130.51 \pm 11.34$ & 4.01 & $130.33 \pm 13.44$ & 3.87 & $133.41 \pm 12.62$ & 6.32 \\
\hline
\end{tabular}

GE, Grupo Experimental; GC, Grupo Controle; EDT, Efeito da Dupla Tarefa; cDTN, com dupla tarefa (stroop test número); CDTC, com dupla tarefa (stroop test cor); CDTCN, com dupla tarefa (stroop test cor número). a , diferença estatisticamente significante na cDTN, entre o GE e o GC; $b$, diferença estatisticamente significante na CDTC, entre o GE e o GC; $(p<0.05)$.

\section{Discussão}

O estudo avaliou os efeitos da dupla tarefa na marcha em esteira de indivíduos com DP, por meio do Stroop Test adaptado para indivíduos analfabetos, e foram encontrados resultados que demonstram que a execução de tarefas duplas (motora-cognitiva) por indivíduos com DP interferem no desempenho desses sujeitos, onde foi observado que a tarefa motora é priorizada. 
Os primeiros achados de nosso estudo apontam que as amplitudes articulares não sofreram interferências das condições de dupla tarefa nos dois grupos estudados. Mesmo, quando consideramos que os participantes com DP apresentaram uma maior flexão do joelho (isto é, uma diminuição da amplitude mínima e máxima) que os indivíduos controle, nas condições que requerem mais atenção. Talvez estes achados possam ser explicados pelos padrões posturais adaptativos das pessoas com DP, que promovem uma postura flexora dos membros inferiores e do tronco ${ }^{19}$.

Esperávamos no presente estudo, que à medida que as demandas atencionais nas condições de dupla tarefa aumentassem, uma maior flexão do joelho seria encontrada. Entretanto, não observamos esse fato. O que pode ser decorrente do fato, de a marcha em esteira em pacientes com DP melhorar a estabilidade e segurança articular, e diminuir a alta taxa de cocontração $0^{20}$, por meio de uma redução da coativação de flexores (bíceps femoral) e extensores (vasto lateral) do joelho e tornozelo de pacientes com DP, mas não nos controles, sugerindo um efeito específico da esteira na DP20.

O presente estudo, também observou a dificuldade dos indivíduos em alternar a atenção entre vários estímulos, a fim de dificultar o processamento de informações motoras e cognitivas de forma simultânea. Nesta situação, deslocar a atenção de uma informação motora (marcha em esteira) para outra tarefa, no caso cognitiva (stroop test adaptado), ou vice-versa. Os resultados apresentados nos fazem acreditar que os indivíduos com DP e controles, priorizaram a função motora. Já que as alterações nas atividades cognitivas foram as mais prejudicadas, como podemos observar na tabela 2. Embora apenas no grupo com pacientes controle tenha ocorrido mudanças significativas nas várias condições experimentais aplicadas, podemos observar também uma diminuição no número de acertos no grupo composto pelos participantes com DP. Isto vai de acordo com estudo prévio, onde indivíduos com DP priorizaram a atividade primária, no caso a marcha em esteira ${ }^{21}$. Com relação ao grupo composto por indivíduos com DP, levantamos a hipótese que não houve diferença significativa durante as condições de dupla tarefa, por tratar-se um grupo pequeno de participantes que estavam no estágio inicial da doença.
Porém, em estudos mais recentes foram encontrados resultados diferentes, onde os indivíduos com DP priorizaram a tarefa cognitiva ao invés da manutenção da tarefa motora desempenhada. Esta discrepância entre os achados pode ser explicada pelo paradigma de dupla tarefa onde a interferência depende do tipo de tarefa motora empregada ${ }^{6}$. Além disso, a marcha não é mais considerada apenas uma atividade motora automatizada ${ }^{6}$, e nos pacientes com DP parece existir uma maior ativação durante a caminhada habitual no solo, do que em adultos saudáveis e com idade mais avançada, demonstrando também uma maior atividade cerebral durante condições complexas de caminhada, por exemplo, durante a marcha com obstáculos 22,23 . Dessa forma, outros estudos ${ }^{24}$ sugerem que as estratégias de controle cognitivo podem diferir entre as modalidades de marcha (no solo e na esteira), e que a influência da modalidade no controle cognitivo deve ser considerada na interpretação dos efeitos da dupla tarefa.

Percebe-se assim, a existência de uma relação direta entre a cognição e a marcha, onde os processos cognitivos estão diretamente relacionados com a locomoção e assim, a interferência da dupla tarefa pode levar a prejuízos no desempenho motor. Consequentemente, a escolha de qual interferência cognitiva, deve ser ofertada no treinamento de marcha na esteira parece ter mais relevância clínica. Como podemos observar nos dados relativos as condições de DT, em que a cDTC apresentou o mesmo prejuízo que a cDTCN no número de acertos, quando esperávamos que a cDTCN fosse a que apresenta-se maior demanda atencional, e consequentemente a que o maior número de erros. Esse fato pode ser em parte, reflexo da utilização do protocolo adaptado para analfabetos do Stroop test.

Com relação aos dados clínicos mensurados pela TUG e DGI, observou-se uma diferença estatística significante entre os grupos estudados, com o GC apresentando melhores resultados. Este resultado já era esperado, tendo em vista que na DP há comprometimentos motores, como bradicnesia, freezing, entre outros, que levam a alterações no padrão locomotor dos pacientes com DP, comparado ao grupo controle.

Além disso, na DP existe um déficit cognitivo que também interfere diretamente no desempenho motor. Assim, o menor desempenho do grupo experimental no TUG, vai de encontro com os resultados 
prévios, onde foi encontrado que as variáveis de sexo ou idade são consideradas juntamente com o $\mathrm{MCl}$ (comprometimento cognitivo leve), onde parece intervir como moderador do desempenho do TUG, sugerindo assim que o $\mathrm{MCl}$ tem influência no teste, porém não como uma variável isolada ${ }^{25}$. Outros estudos, também encontraram evidências de uma forte relação entre função cognitiva e as anormalidade da marcha, isto porque eles acreditam que a marcha envolve diversos domínios cognitivos, como função executiva - atencional, habilidades visuoespaciais e até mesmo os recursos de memória6.

É importante mencionar que não foram produzidos cálculos do Efeito da dupla tarefa na variável número de acertos, pois não foi obtido esta variável na condição de tarefa única do Stroop Test. Mas acreditamos, ser interessante investigar se o número de acertos se manteria ou aumentaria quando os indivíduos fossem colocados apenas realizando o Stroop Test, já que em todas as condições, a interferência cognitiva foi associada à interferência motora.

Acreditamos também, que o treino de marcha em esteira com dupla tarefa pode ser mais uma opção no treinamento da função locomotora, além de propiciar um treino de marcha com dupla tarefa com segurança para com pacientes com DP. Desta forma, consideramos de extrema importância um maior aprofundamento deste tema em trabalhos futuros, tendo em vista que essas variáveis estão diretamente relacionadas com o desempenho da marcha e os resultados deste estudo.

Este estudo analisou os efeitos da dupla tarefa na marcha de indivíduos com DP, avaliando o número de acertos do Stroop Test associado à tarefa dupla, com isso acreditamos ser relevante o desenvolvimento de novos estudos investigando a relação entre o número de acertos no Stroop Test quando os indivíduos não fossem expostos a mais de uma tarefa, já que todas as nossas variáveis consideravam a interferência cognitiva e motora simultaneamente. Além disso, como limitação do estudo destaca-se o pequeno número de participantes, onde talvez os resultados encontrados não representem de forma ampla toda a população alvo.

\section{Conclusão}

Os resultados sustentam a hipótese de que a dupla tarefa motora-cognitiva associada à marcha em esteira em indivíduos com DP interfere no desempenho desses indivíduos. Os achados relevam que há um maior comprometimento na função cognitiva, quando comparado a função motora da marcha em esteira, visto que os resultados (números de acertos) da tarefa cognitiva foram os mais prejudicados.

\section{Agradecimentos}

Os autores gostariam de agradecer as contribuições dadas pelos integrantes do grupo de pesquisa em Motricidade e Fisiologia Humana (MOFISH) da Universidade Federal do Rio Grande do Norte - Faculdade de Ciências da Saúde do Trairi.

\section{Contribuições dos autores}

Araújo FR, Araújo DS e Cacho EWA participaram da concepção, delineamento, coleta de dados da pesquisa, análise estatística dos dados da pesquisa, interpretação dos resultados e redação do artigo científico. Gomes CLA participou da análise estatística dos dados da pesquisa, interpretação dos resultados e redação do artigo científico. Medeiros ALS participou da análise estatística dos dados da pesquisa e redação do artigo científico. Gondim ALM participou da coleta de dados da pesquisa. Cacho RO participou da interpretação dos resultados e redação do artigo científico.

\section{Conflitos de interesses}

Nenhum conflito financeiro, legal ou político envolvendo terceiros (governo, empresas e fundações privadas, etc.) foi declarado para nenhum aspecto do trabalho submetido (incluindo, mas não se limitando a subvenções e financiamentos, participação em conselho consultivo, desenho de estudo, preparação de manuscrito, análise estatística, etc.).

\section{Referências}

1. Capato TTC, Domingos JMM, Almeida LRS. Versão em Português da diretriz Europeia de Fisioterapia para a Doença de Parkinson. [Internet]. 2015. Dispoível em: https://abrafin.org.br/versao-emportugues-da-diretriz-europeia-de-fisioterapia-para-a-doenca-deparkinson/

2. Nonnekes J, Růžička E, Nieuwboer A, Hallett M, Fasano A, Bloem BR. Compensation Strategies for Gait Impairments in Parkinson Disease A Review. JAMA Neurol. 2019;76(6):718-725. doi: 10.1001/ jamaneurol.2019.0033 
3. Ni M, Hazzard JB, Signorile JF, Luca C. Exercise Guidelines for Gait Function in Parkinson's Disease: A Systematic Review and Meta-analysis. Neurorehabilitation and Neural Repair 2018;32(10):872-86. doi: 10.1177/1545968318801558

4. Al-Yahya E, Mahmoud W, Meester D, Esser P, Dawes H. Neural Substrates of Cognitive Motor Interference During Walking; Peripheral and Central Mechanisms. Front in Hum Neurosci. 2019;12(1):536. doi: 10.3389/fnhum.2018.00536

5. Howcroft J, Kofman J, Lemaire ED, Mcilory WE. Analysis of dualtask elderly gait in fallers and non-fallers using wearable sensors. J Biomech. 2016;(49):992-1001. doi: 10.1016/j.jbiomech.2016.01.015

6. Bello O, Marquez G, Fernandez-Del-Olmo M. Effect of Treadmill Walking on Leg Muscle Activation in Parkinson's Disease. Rejuvenation Res. 2019;22(1):71-78. doi: 10.1089/rej.2018.2084

7. Hsiu-Chen C, Chiung-Chu C, Jiunn-Woei L, Wei-Da C, Xin WY, Ya-Ju C et al. The effects of dual - task in patients with Parkinson'S disease performing cognitive-motor paradigms. J Clin Neurosci. 2020;72:72-8. doi: 10.1016/j.jocn.2020.01.024

8. Wu T, Hallett M, Chan P. Automaticidade motora na doença de Parkinson. Neurobiol Dis. 2015;82:226-34. doi: 10.1016/j. nbd.2015.06.014

9. Wu T, Liu J, Zhang H, Hallett M, Zheng Z, Chan P. Atenção aos movimentos automáticos na doença de Parkinson: modo automático modificado no estriado. Cereb Cortex. 2015;25(10):3330-42. doi: 10.1093/cercor/bhu135

10. Yogev-Seligmann G, Hausdorff JM, Giladi N. O papel da função executiva e da atenção na marcha. Mov Disord. 2008;23:329-42. doi: $10.1002 / \mathrm{mds} .21720$

11. Agner S, Bernet J, Brulhart $Y$, Radlinger L, Rogan S. Spatiotemporal gait parameters during dual task walking in need of care elderly and young adults. Z Gerontol Geriatr. 2015;48(8):740-6. doi: $10.1007 / \mathrm{s} 00391-015-0884-1$

12. Kleiner AFR, Pagnussat AS, Prisco GD,Vagnini A, Stocchi $\mathrm{F}$, Pandis MF et al. Analyzing gait variability and dual-task interference in patients with Parkinson's disease and freezing by means of the word-color Stroop test. Aging Clin Exp Res. 2018;30(9):1137-1142. doi: 10.1007/s40520-017-0862-0

13. Fahn S, Elton RL, and members of the UPDRS Development Committee. Unified Parkinson's Disease Rating Scale. In: Fahn S, Marsden CD, Calne DB, Goldstein M. Recent developments in Parkinson's disease. Florham Park [N], USA]: Macmillan Healthcare Information; 1987. p.153-63.

14. Schenkman ML, Clark K, Xie T, Kuchibhatla M, Shinberg M, Ray L. Spinal movement and performance of a standing reach task in participants with and without Parkinson disease. Physical Therapy. 2001;81(8):1400-11. doi: 10.1093/ptj/81.8.1400
15. Karuka AH, Silva JAMG, Navega MT. Análise da concordância entre instrumentos de avaliação do equilíbrio corporal em idosos. Rev Bras Fisioter. 2011;15(6):460-6. doi: 10.1590/S141335552011000600006

16. Souza RF, Gazzola JM, Gananca MM, Paulino CA. Correlation between the body balance and functional capacity from elderly with chronic vestibular disorders. Braz J Otorhinolaryngol. 2011;77(6):791-8

17. Castro SM, Perracini MR, Ganança FF. Versão brasileira do Dynamic Gait Index. Rev Bras Otorrinolaringol. 2006;72(6):817-25. doi: 10.1590/S0034-72992006000600014

18. Kulayf T. O teste de cores e palavras de Stroop modificado para analfabetos. [dissertação]. São Paulo: Instituto de Psicologia da Universidade de São Paulo; 2005.

19. Kelly VE, Janke AA, Shumway-Cook A. Effects of instructed focus and task difficulty on concurrent walking and cognitive task performance in healthy young adults. Exp Brain Res. 2010;207(12):65-73. doi: $10.1007 / \mathrm{s} 00221-010-2429-6$

20. Bello O, Marquez G, Camblor M, Fernandez-Del-Olmo $M$. Mechanisms involved in treadmill walking improvements in Parkinson's disease. Gait Posture. 2010;32(1):118-23. doi: 10.1016/j.gaitpost.2010.04.015

21. Plummer-D'Amato $P$, Altmann LJ, Behrman AL, Marsiske M. Interference between cognition, double-limb support, and swing during gait in community-dwelling individuals poststroke. Neurorehabil Neural Repair. 2010;24(6):542-9. doi: 10.1177/1545968309357926

22. Peterson DS, Pickett KA, Duncan R, Perlmutter J, Earhart GM. Gait-Related Brain Activity in People with Parkinson Disease with Freezing of Gait. PLoS ONE. 2014;9(3):e90634. doi: 10.1371/ journal.pone.0090634

23. Maidan I, Rosenberg-Katz K, Jacob Y, Giladi N, Deutsch J, Hausdorff J et al. Ativação cerebral alterada em condições complexas de caminhada em pacientes com doença de Parkinson. Transtorno relacionado ao parkinsonismo. 2016;25:916. doi: 10.1016/j.parkreldis.2016.01.025

24. Wrightson JG, Smeeton NJ. Walking modality, but not task difficulty, influences the control of dual-task walking. Gait \& Posture. 2017;(58):136-138. doi: 10.1016/j.gaitpost.2017.07.042

25. Ibrahim A, Singh DKA, Shahar S. 'Timed Up and Go' test: Age, gender and cognitive impairment stratified normative values of older adults. PLoS One. 2017;12 (10):e0185641. doi: 10.1371/ journal.pone.0185641 